\title{
Automated attendance system using Markov random field algorithm
}

\author{
Abhilash Maradwar ${ }^{1}$, Nikita Suryawanshi ${ }^{2}$, Tushar Panpaliya ${ }^{3}$, Suraj Songire $^{4}$ \\ PVG's COET, PUNE, India ${ }^{1,2,3,4}$
}

\begin{abstract}
Computer dependent communication is based on a very important factor, which is Authentication. Human face recognition is an important branch of biometric verification and has a wide range of application. In this paper we describe a method for Automated Attendance System which is based on face recognition by Markov Random Field algorithm. The system describes a method for marking the student's attendance using proposed algorithm and also provides additional features like maintaining the student database and required information about them. There are many advantages of this system over traditional method for attendance. It eliminates the overhead of manually marking the attendance and reduces the time and efforts. Markov Random Field algorithm is Pose-Invariant that does not require manually selection of facial landmarks or head pose estimation. In order to improve the performance of our pose normalization method in face recognition, we also present an algorithm for classifying whether a given face image is at a frontal or non-frontal pose. Experimental results on different datasets are presented to demonstrate the effectiveness of the proposed approach
\end{abstract}

Keywords: Markov Random Field, Pose-Invariant, frontal or non-frontal pose

\section{INTRODUCTION}

Student's attendance records are important documents for academic institutions that reflect the students' performances and the credibility of the academic institutions. Nevertheless, the process of managing these documents had been done manually, by pen and papers thus making it less efficient. This paper discusses about the development of the new Online Attendance System as the enhancement of the previous developed system named Attendance Register System for higher academic institutions. The new online system is being developed by adapting the concept of the web-based system architecture. The web-based system architecture used in the development of this system involves integration of various open source web-based technologies such as MySQL, PHP and Apache Web Server hence making the Online Attendance System more efficient and cost effective. The development of the Online Attendance System involves four main phases which are the construction of the conceptual framework where the scopes and requirements as well as the target users of the system had been identified, developing the proposed system's architecture by adapting to the web-based architecture, and the construction of the logical design and the physical design. The Online Attendance System consists of automated processes such as able to generate online attendance reports, warning letters and graph charts thus making the processes of recording and reporting the students' attendances more efficient and well-organized.

With the rapid development in the field of pattern recognition and its uses in different areas e.g. (signature recognition, facial recognition), arises the importance of the utilization of this technology in different areas in large organizations. This is mainly because these applications help the top-management take decisions that improve the performance and effectiveness of the organization. On the other hand, for an organization to be effective, it needs accurate and fast means of recording the performance of the people inside this organization. Biometric recognition has the potential to become an irreplaceable part of many identification systems used for evaluating the performance of those people working within the organization. Although biometric technologies are being applied in many fields it has not yet delivered its promise of guaranteeing automatic human recognition. This research is the first of its kind to attempt to provide an automated attendance system that recognizes students using face recognition technology through an image/video stream to record their attendance in lectures or sections and evaluating their performance accordingly.

We have studied the literature available in the fields of pattern recognition and biometric systems with a focus on facial recognition. In addition, a study of previous attendance systems and it atomization attempts was conducted to examine the similar previous systems. The literature is divided into three main parts; the first part examines the different biometric characteristics and systems. After that, an in depth study on facial recognition is conducted for it is the technology to be used by our proposed project. The final portion of this literature review presents the different time and attendance systems offered by different researchers and vendors.

\section{MOTIVATION}

Student's attendance records are important documents that reflect their performance.Traditional attendance register system is time consuming. It involved one by one marking the attendance of the students by calling their name and check whether the student is present or not. Other method was to supply the attendance sheet to student to mark their attendance which have high chances of proxies. Thus we introduce a system which is based on Multi-face 
recognition that will reduce the proxies and also time to Step 1: Gather and prepare the data.

mark the attendance.This will also reduce the need to Let the training set of face images be I1, I2... IM

reduce paper pen work for managing documents.

Step 2: Find optimal set of local warps.

Biometric systems over normal automated system is We have two images, the Training image $I$ and the gallery preferred to authenticate the user.Real human physical image $T$. The images are divided into $M$ blocks (rectangle characteristics are almost impossible to change.Passwords, cards are subjected to theft, loss or passing to someone else.

\section{OVERVIEW}

1) Camera: Camera is used to capture face image of students in which Image containing facewill be used for detection and recognition.

2) Internal Resize: As input will be some fixed size face images. So before using for recognition, images should be resized so that it can be as per the input face image.

3) Face Identification and feature extraction: In face detection, particular face region will be considered and features will be extracted using suitable feature extraction algorithm.

4) Save template in DB: If input face image will be new then it should be stored in DB. So that further that image will be used in matching process. Automated attendance system using face recognition.

5) Match with template in DB: If face image is already present in DB then for making an attendance it will first match with images in database. And if match found the attendance will be marked.

6) Displayed in Application: After detection and matching if input image found in database then it will be displayed on output screen i.e. in application as an attendance will be marked.

\section{SYSTEM IMPLEMENTATION}

A. Algorithms Used :

Here, lattice points whose local patches are inside the image form a set of MRF nodes $V$. The set of warps $P i$ can be considered as the set of possible labels for node $i$. A 4connected neighbourhood system is then by edges $E$ of the MRF. We also present a method that is classifies whether an input face image is frontal or non-frontal. This method extracts dense SIFT descriptors from the input face image and performs classification using the Support Vector Machine (SVM) algorithm.

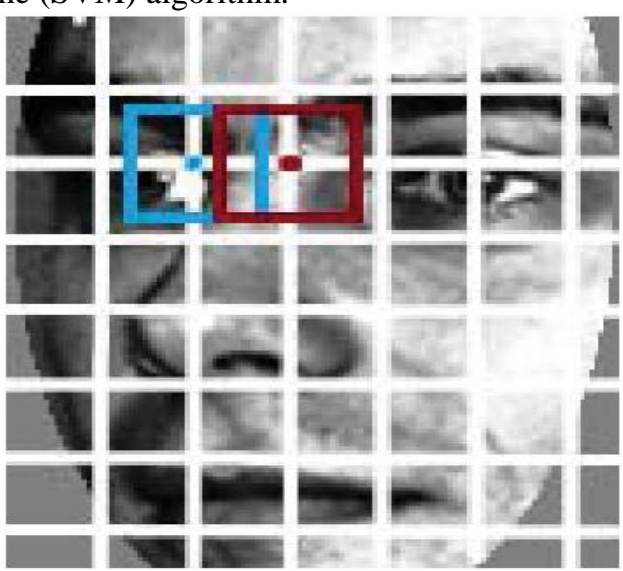

Fig. 1. Two neighboring MRF nodes with overlapping patches

Fig1: Neighbouring MRF nodes patches) and for each pair of corresponding patches, $I i$ and $T i$, a local warp $W i$ is estimated. The warp Wi, parameterized by the vector $\mathbf{p} i$, minimizes the error function. If $W(\mathbf{p})$ is an affine warp with parameters $\mathbf{p}=$ $(p 1, p 2, p 3, p 4, p 5, p 6) T$, it can be written as

$$
W(\mathbf{p})=\left(\begin{array}{ccc}
1+p_{1} & p_{3} & p_{5} \\
p_{2} & 1+p_{4} & p_{6}
\end{array}\right) .
$$

Step 3: Find Optimal Labels using BP algorithm.

It is an inference technique that works by passing local messages along the nodes of a MRF [2]. Using negative logarithmic probabilities, a message from node $i$ to

Node $j$ at time $t$ is defined as

$$
\begin{gathered}
m_{i j}^{t}\left(\mathbf{p}_{i}\right)=\min _{\mathbf{p}_{i} \in \mathcal{P}_{i}}\left\{E_{i}\left(\mathbf{p}_{i}\right)+\lambda E_{i j}\left(\mathbf{p}_{i}, \mathbf{p}_{j}\right)+\right. \\
\left.\sum_{k: k \neq j,(k, i) \in \mathcal{E}} m_{k i}^{t-1}\left(\mathbf{p}_{i}\right)\right\} .
\end{gathered}
$$

Assume that all messages converge after s iterations, the belief of node $\mathrm{i}$ for $\mathrm{pi} \cdot \mathrm{Pi}$, bi (pi ) is computed as

$$
b_{i}\left(\mathbf{p}_{i}\right)=-E_{i}\left(\mathbf{p}_{i}\right)-\sum_{k:(k, i) \in \mathcal{E}} m_{k i}^{s}\left(\mathbf{p}_{i}\right) .
$$

Step 4: Frontal View Classification.

It is important to be able to automatically decide if the input face image is frontal or non-frontal. In our approach, the frontal-view classification is performed using the Support Vector Machine (SVM) algorithm. First, dense Scale Invariant Feature Transform (SIFT) descriptors are extracted from image grid points in order to obtain a representation that is robust to noise and illumination variations. The dimension of the concatenated descriptor vector is reduced for efficient processing by using Random Projection (RP). Finally, an SVM is employed to decide whether the face image is at the frontal pose or not.

B.Input and Output:

The inputs of our system consist of bit streams representing the image, a face, and a set of projections. The image, as well as the face, will consist of $122 \times 92$ $=10304$ pixels, each being 8-bit grayscale (256 levels ranging from 0 to 255). These values were used successfully by many research groups. These figures were chosen because they provide a good balance between size and accuracy. The output of our system will consist of the face ID with the closest match. 


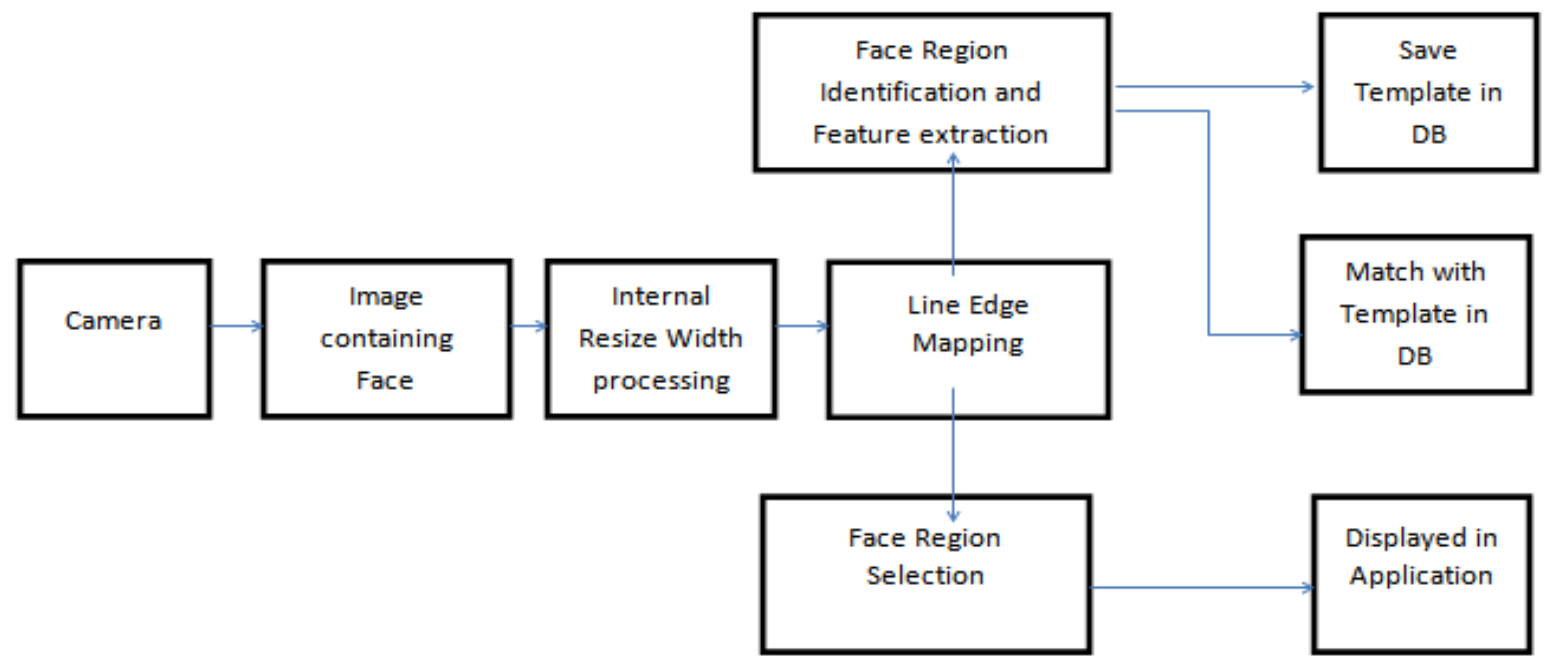

Fig2:System Overview

All this information will be displayed on the GUI of the PC. It will always call a stored procedure in the database with image ID as its parameter to record the student's attendance.

\section{EXPERIMENTAL RESULTS}

\section{A. Frontal-View Classification Using Dense SIFT Descriptors}

The proposed frontal-view classification algorithm was trained using an SVM on 2D images generated from the 3D faces in the USF 3D database [7]. By rotating the 3D models and projecting them into the image plane, we can synthesize the $2 \mathrm{D}$ face images at different viewing angles. Face images with less than $\pm 5^{\circ}$ in both the yaw and pitch angles are labelled as frontal [4]. 2D face images of a person in the database generated at different poses and the visualization of their corresponding dense SIFT descriptors. As the USF 3D database contains the geometry as well as the texture information of the 3D faces, the face images at different illumination conditions [1][8] can also be generated from the surface normals and albedo.

This is necessary in order for the methodto handle possible illumination variations in the test images. We tested the proposed frontal-view classification algorithm on four different databases including the USF 3D database,FERET, CMU-PIE, and Multi-PIE.

For the USF 3D database, the synthesized face images were divided into five subsets. Four of them were used for training and the remaining subset was used for testing. It takes less than 4 seconds to perform the frontal-view classification for aninput face image of size $130 \times 150$ on an Intel Xeon $2.13 \mathrm{GHz}$ desktop.

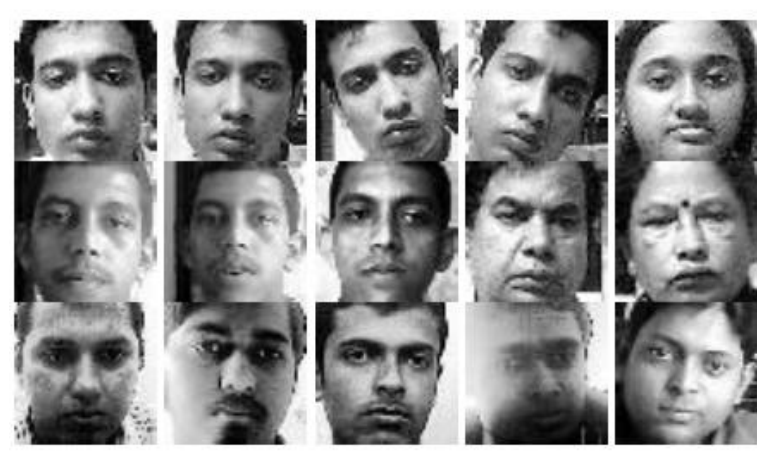

Fig3: Training Images

TABLE 2: FACE DETECTION AND RECOGNITION RATE

\begin{tabular}{|c|c|c|}
\hline Face Orientations & Detection Rate & Recognition Rate \\
\hline $\mathrm{O}^{\circ}$ (Frontal face) & $98.7 \%$ & $95 \%$ \\
\hline $18^{\circ}$ & $80.0 \%$ & $78 \%$ \\
\hline $54^{\circ}$ & $59.2 \%$ & $58 \%$ \\
\hline $72^{\circ}$ & $0.00 \%$ & $0.00 \%$ \\
\hline $90^{\circ}$ (Profile face) & $0.00 \%$ & $0.00 \%$ \\
\hline
\end{tabular}

Fig 4 Table

We performed a set of experiments to demonstrate the efficiency of the proposed method. 30 different images of 10 persons are used in training set. A sample binary image detected by the Extract Face function using Paul-Viola Face extracting Frame work detection method. From table 2 it is been observed that with the increasing of record their time of entry and exit, the authors proposed the attendance management system based on face recognitiontechnology in the institutions/organizations. The system takes attendance of each student by continuous observation at the entry and exit points. The result of our preliminary experiment shows improved performance in the estimation of the attendance compared to the traditional black and white attendance systems. In further work, authors intend to improve facerecognition 
effectiveness by using the interaction among our system[5][6], the users and the administrators. On the other hand, our system can be used in a completely new dimension of

face recognition application, mobile based face recognition, which can be an aid for common people to know about any person being photographed by cell phone camera includingproper authorization for accessing a centralized database face angle with respect to camera face detection and recognition rate is become decreases.

\section{VI.CONCLUSION}

Here in this paper, we presented a method for synthesizing the virtual frontal view from a non- frontal face image, by dividing the input image into overlapping patches, a globally optimalset of local warps can be estimated to transform the patches record their time of entry and exit.

The system takes attendance of each student by continuous observation at the entry and exit points. The result of our preliminary experiment shows improved performance in the estimation of the attendance compared to the traditional black and white attendance systems. Current work is focused on theface detection algorithms from images or video frames. In further work, authors intend to improve face recognition effectiveness by using the interaction among our system, the users and the administrators. On the other hand, our system can be used in a completely new dimension of face recognition application, mobile based face recognition, which can be an aid for common people to know about any person being photographed by cell phone camera including proper authorization for accessing a centralized database.

\section{REFERENCES}

1. G. Hager and P. Belhumeur, "Efficient region tracking with parametric Models of geometry and illumination," IEEE Trans. Pattern Anal. Mach. Intel, vol. 20, no. 10, pp. 1025-1039, Oct. 1998.

2. N. Komodakis and G. Tziritas, "Image completion using efficient Belief Propagation via priority scheduling and dynamic pruning," IEEE Trans. Image Process, vol. 16, no. 11, pp. 2649-2661, Nov. 2007.

3. "Study of Implementing Automated Attendance System Using Face Recognition Technique" International Journal of Computer and Communication Engineering, Vol. 1, No. 2, July 2012.

4. C. Burges, "A tutorial on support vector machines for pattern recognition,” Data Min. Knowl. Discov, vol. 2, no. 2, pp. 121-167, 1998.

5. T. Tian, T. Kanade, and J. Cohn, "Recognizing Action Units for Facial Expression Analysis," IEEE Trans. Pattern Analysis and Machine Intelligence, vol. 23, no. 2, pp. 97-115, Feb. 2001.

6. B. Fasel and J. Luettin, "Automatic Facial Expression Analysis: A Survey," Pattern Recognition, vol. 36, no. 1, pp. 259-275, 2003.

7. A. Shashua and T. Riklin-Raviv, "The Quotient Image: Class Based Re-Rendering and Recognition with Varying Illuminations," IEEE Trans. Pattern Analysis and Machine Intelligence, vol. 23, no. 2, pp. 129-139, Feb. 2001.

8. X. Xie and K. Lam, "Face Recognition under Varying Illumination Based on a 2D Face Shape Model," Pattern Recognition, vol. 38, no. 2, pp. 221-230, 2005. 